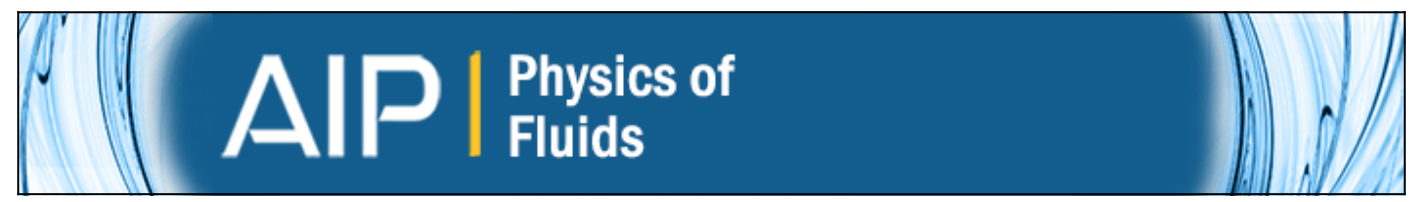

\title{
Oxygen transport properties estimation by classical trajectory-direct simulation Monte Carlo
}

Domenico Bruno, Aldo Frezzotti, and Gian Pietro Ghiroldi

Citation: Physics of Fluids (1994-present) 27, 057101 (2015); doi: 10.1063/1.4921157

View online: http://dx.doi.org/10.1063/1.4921157

View Table of Contents: http://scitation.aip.org/content/aip/journal/pof2/27/5?ver=pdfcov

Published by the AIP Publishing

\section{Articles you may be interested in}

Application of Gaussian random field theory to direct simulation of rarefied gas flow near rough surface

AIP Conf. Proc. 1501, 1160 (2012); 10.1063/1.4769672

Consistent treatment of transport properties for five-species air direct simulation Monte Carlo/Navier-Stokes applications

Phys. Fluids 24, 077101 (2012); 10.1063/1.4729610

Introduction to MOLFLOW+: New graphical processing unit-based Monte Carlo code for simulating molecular flows and for calculating angular coefficients in the compute unified device architecture environment

J. Vac. Sci. Technol. A 27, 1017 (2009); 10.1116/1.3153280

An approach for simulating the transport of spherical particles in a rarefied gas flow via the direct simulation Monte Carlo method

Phys. Fluids 13, 3482 (2001); 10.1063/1.1409367

Direct simulation Monte Carlo study of orifice flow

AIP Conf. Proc. 585, 924 (2001); 10.1063/1.1407658

\section{How to Simulate \&} Design Microfluidics

\section{Devices}

$\triangle$ COMSOL
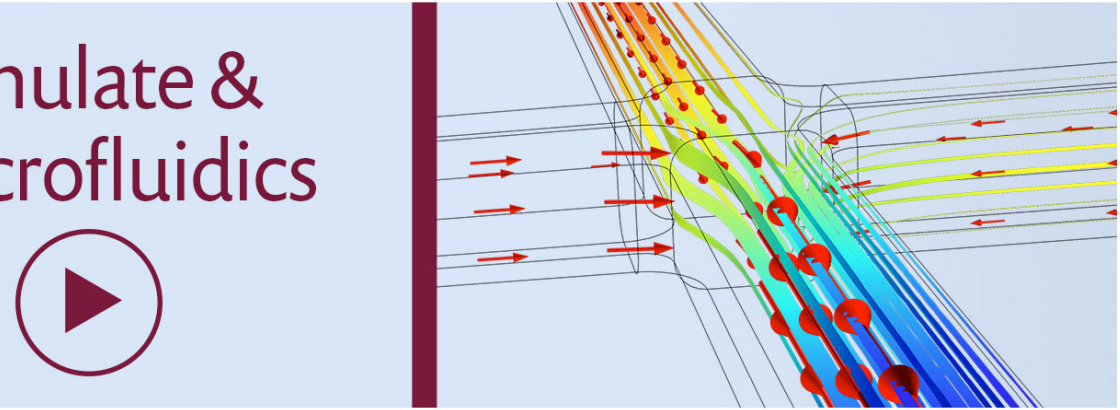


\title{
Oxygen transport properties estimation by classical trajectory-direct simulation Monte Carlo
}

\author{
Domenico Bruno, ${ }^{1, a)}$ Aldo Frezzotti, ${ }^{2, b)}$ and Gian Pietro Ghiroldi²,c) \\ ${ }^{1}$ Istituto di Metodologie Inorganiche e dei Plasmi, Consiglio Nazionale delle Ricerche- \\ Via G. Amendola 122, 70125 Bari, Italy \\ ${ }^{2}$ Dipartimento di Scienze e Tecnologie Aerospaziali, Politecnico di Milano-Via La Masa \\ 34, 20156 Milano, Italy
}

(Received 16 January 2015; accepted 4 May 2015; published online 19 May 2015)

\begin{abstract}
Coupling direct simulation Monte Carlo (DSMC) simulations with classical trajectory calculations is a powerful tool to improve predictive capabilities of computational dilute gas dynamics. The considerable increase in computational effort outlined in early applications of the method can be compensated by running simulations on massively parallel computers. In particular, Graphics Processing Unit acceleration has been found quite effective in reducing computing time of classical trajectory (CT)-DSMC simulations. The aim of the present work is to study dilute molecular oxygen flows by modeling binary collisions, in the rigid rotor approximation, through an accurate Potential Energy Surface (PES), obtained by molecular beams scattering. The PES accuracy is assessed by calculating molecular oxygen transport properties by different equilibrium and non-equilibrium CT-DSMC based simulations that provide close values of the transport properties. Comparisons with available experimental data are presented and discussed in the temperature range 300-900 K, where vibrational degrees of freedom are expected to play a limited (but not always negligible) role. (C) 2015 AIP Publishing LLC. [http://dx.doi.org/10.1063/1.4921157]
\end{abstract}

\section{INTRODUCTION}

In the study of dilute gases, the dynamics of binary encounters plays a key role by determining the relationships between pre- and post-collision molecular states. ${ }^{1}$ When monatomic gases are considered, collision dynamics is largely amenable to analytical treatment ${ }^{2}$ which allows obtaining scattering patterns with modest numerical effort, even for realistic interatomic potentials. ${ }^{3}$ Molecular collision dynamics is definitely more complex because of the larger number of involved degrees of freedom and their coupling. Early simplified mechanical models of translational-rotational coupling (rough spheres, loaded spheres, spherocylinders) ${ }^{4}$ were mainly used for transport properties' estimations but proved to be not flexible enough to fit experimental data. Since the advent of high speed computers and rarefied gas simulations based on Direct Simulation Monte Carlo (DSMC), ${ }^{5}$ the study of non-equilibrium gas flows involving polyatomic species has been mainly based on phenomenological models, originating from the Borgnakke-Larsen model. ${ }^{6}$ Such models ignore the detailed dynamics of binary molecular encounters, directly connecting pre- and post-collision molecular states through relatively simple and computationally inexpensive relationships. Moreover, they contain physical parameters that allow tuning total collision cross sections and intensities of rotational-translational coupling in order to match experimental data. ${ }^{7}$

Deeper insight into the dynamics of molecular collisions and greater model predictive capabilities are provided by Classical Trajectory (CT) calculations, in which the motion of two colliding molecules is deterministically computed from classical mechanics by specifying a potential energy surface (PES) $V$. The function $V$, depending on the relative position and orientation of the

\footnotetext{
a)Electronic mail: domenico.bruno@cnr.it

b) Electronic mail: aldo.frezzotti@polimi.it

c)Electronic mail: gpghiro@gmail.com
} 
molecules as well as on any additional coordinate characterizing internal molecular configurations, can be determined by molecular beams scattering experiments ${ }^{8}$ or from quantum $a b$ initio calculations. ${ }^{9}$ Most CT technique applications are focused on obtaining transport properties whose general expressions are derived from Chapman-Enskog expansions. ${ }^{10,11}$ Although early applications are limited to diatomic linear molecules, ${ }^{12}$ more recent ones consider non-linear polar and non-polar molecules ${ }^{13,14}$ as well as atom-molecule interactions. ${ }^{15}$ Combining CT techniques with particle schemes for the numerical solution of the Boltzmann equation ${ }^{1}$ allows modeling non-equilibrium processes beyond the hydrodynamic regime. Shock wave propagation and relaxation processes in nitrogen have been studied by Koura in a series of papers ${ }^{16,17}$ based on a hybrid numerical scheme in which DSMC creates the collisions queue and pre-collision states for each colliding pair. Post-collision states, used by DSMC to update the system evolution, are obtained by integrating Newton or Hamilton equations describing two-body interaction for each collision. Although the results were quite satisfactory, it was observed that a typical hybrid CT-DSMC simulation was two orders of magnitude slower than the corresponding DSMC simulation based on a phenomenological collision model. As already suggested in Ref. 16, parallel computing techniques can alleviate the higher computational load of CT-DSMC simulations. Actually, a careful organization of the collisions sequence in a DSMC time step allows the concurrent calculation of all binary collision trajectories required by a DSMC time step. The massively parallel architecture of modern Graphics Processing Units (GPUs) has been effectively exploited to reduce the computational overhead of CT-DSMC simulations in Refs. 18 and 19, where rotational relaxation dynamics in nitrogen and oxygen has been studied.

Considering that the methodological and computational frameworks of CT and CT-DSMC techniques are rather clearly established, the present work aims at assessing the accuracy of a specific PES, describing the interaction of $\mathrm{O}_{2}$ molecules in the rigid rotor approximation. The PES structure, described in Ref. 8, has been determined from careful and accurate molecular beams scattering experiments but, to the authors' knowledge, it has not yet been used to obtain gas flows properties' predictions. PES quality has been assessed by computing $O_{2}$ transport properties: shear and bulk viscosities, thermal conductivity, and self-diffusion coefficient. The obtained results have been compared with available experimental data in the temperature range 300-900 K, where the presence of vibrational modes has a limited (but not always negligible) effect on $\mathrm{O}_{2}$ transport properties. In order to increase confidence on numerical methods and results, the same transport property has been obtained from different implementations of CT and CT-DSMC calculations, when possible. Table I summarizes the different techniques adopted to estimate $\mathrm{O}_{2}$ transport properties.

In accordance with the aims of the work and adopted methods, Secs. II-VI describe the structure of the PES and the equations governing the dynamics of $\mathrm{O}_{2}-\mathrm{O}_{2}$ encounters, the CT-DSMC scheme, as it stems from the underlying kinetic equation and the particular implementations listed above. Section VII is devoted to concluding remarks.

\section{II. $\mathrm{O}_{2}-\mathrm{O}_{2}$ POTENTIAL ENERGY SURFACE AND BINARY COLLISION DYNAMICS}

In the present study, oxygen molecules are assumed to behave as classical rigid rotators. The positions and orientations of two colliding molecules, in an arbitrarily defined "laboratory" reference frame, are specified by the center of mass positions $\mathbf{R}_{i}$ and by the vectors $\mathbf{r}_{i}$ specifying the relative position of the $O$ atoms within molecule $i(i=1,2)$. The vectors $\mathbf{r}_{i}$ have constant length $d$, equal to the internuclear $O-O$ distance. The adopted intermolecular potential is taken from Ref. 8 , where the interaction between two oxygen molecules is described by the following truncated spherical harmonic expansion:

$$
\begin{aligned}
V\left(R_{12}, \theta_{1}, \theta_{2}, \phi\right)= & 4 \pi\left\{V^{000}\left(R_{12}\right)+V^{202}\left(R_{12}\right)\left[Y_{20}^{20}\left(\theta_{1}, \theta_{2}, \phi\right)+Y_{02}^{20}\left(\theta_{1}, \theta_{2}, \phi\right)\right]+\right. \\
& \left.V^{220}\left(R_{12}\right) Y_{22}^{00}\left(\theta_{1}, \theta_{2}, \phi\right)+V^{222}\left(R_{12}\right) Y_{22}^{20}\left(\theta_{1}, \theta_{2}, \phi\right)\right\} .
\end{aligned}
$$

As shown in Fig. $1, R_{12}$ is the modulus of the vector $\mathbf{R}_{12}=\mathbf{R}_{2}-\mathbf{R}_{1}$ pointing from the center of mass of molecule 1 to the center of mass of molecule 2 . The angle $\theta_{i}$ is respectively formed by the directions of vectors $\mathbf{r}_{i}$ and $\mathbf{R}_{12}$, which define a plane $\Pi_{i}$ containing molecule $i$ and the centers of 
TABLE I. CT-MC: Direct CT-Monte Carlo evaluation of transport properties, as done in early CT applications. Ref. $12 \mathrm{GK}$ : Green-Kubo formalism Ref. 20 applied to CT-DSMC equilibrium simulations. USF: CT-DSMC non-equilibrium simulations of Uniform Shear Flows. HF: CT-DSMC non-equilibrium simulations of one-dimensional heat flux in $\mathrm{O}_{2}$ confined between parallel plates kept at different temperatures. TPD: Equilibrium CT-DSMC simulations of tagged particles diffusion.

\begin{tabular}{lccccc}
\hline \hline & CT-MC & GK & USF & HF & TPD \\
\hline Shear viscosity $\eta$ & $\times$ & $\times$ & $\times$ & & \\
Thermal conductivity $\lambda$ & $\times$ & $\times$ & & $\times$ & \\
Bulk viscosity $\kappa$ & $\times$ & & & & \\
Self-diffusion coefficient $\mathcal{D}$ & & & & & $\times$ \\
\hline \hline
\end{tabular}

mass of both molecules, if $\theta_{i} \neq 0, \pi$. The angle $\phi$ specifies the relative orientation of the planes $\Pi_{1}$ and $\Pi_{2}$ which, by construction, have the straight line connecting the molecular centers of mass in common. The four radial coefficients of the potential $V^{000}\left(R_{12}\right), V^{202}\left(R_{12}\right), V^{220}\left(R_{12}\right)$, and $V^{222}\left(R_{12}\right)$ are fully described and discussed in the original paper. However, their form is also given in Appendix A, for completeness. The effects of relative molecular orientation are taken into account by the bipolar spherical harmonics $Y_{20}^{20}\left(\theta_{1}, \theta_{2}, \phi\right), Y_{02}^{20}\left(\theta_{1}, \theta_{2}, \phi\right), Y_{22}^{00}\left(\theta_{1}, \theta_{2}, \phi\right)$, and $Y_{22}^{20}\left(\theta_{1}, \theta_{2}, \phi\right) .{ }^{8,21}$ It is to be remarked that the potential parameters setting reported in Appendix A and adopted in this work correspond to the nominal ones. The influence of uncertainties given in Ref. 8 has not been explored. Given the potential energy surface, Eq. (1), the classical equations of motion of the $\mathrm{O}_{2}-\mathrm{O}_{2}$ dimer have been derived. For computational convenience, a change of coordinates has been performed and the interatomic relative position vectors $\mathbf{r}_{1}$ and $\mathbf{r}_{2}$ have been used in place of the angular coordinates $\theta_{1}, \theta_{2}$, and $\phi$. The latter being related to the former by the following relationships:

$$
\tan \theta_{1}=\frac{\left|\mathbf{R}_{12} \wedge \mathbf{r}_{1}\right|}{\mathbf{R}_{12} \cdot \mathbf{r}_{1}}, \quad \tan \theta_{2}=\frac{\left|\mathbf{R}_{12} \wedge \mathbf{r}_{2}\right|}{\mathbf{R}_{12} \cdot \mathbf{r}_{2}}, \quad \tan \phi=\frac{\left|\mathbf{N}_{1} \wedge \mathbf{N}_{2}\right|}{\mathbf{N}_{1} \cdot \mathbf{N}_{2}}
$$

being $\mathbf{N}_{1}=\mathbf{R}_{12} \wedge \mathbf{r}_{1}$ and $\mathbf{N}_{2}=\mathbf{R}_{12} \wedge \mathbf{r}_{2}$. In terms of the new set of variables, the equations of motions become

$$
\begin{aligned}
& m_{O} \ddot{\mathbf{R}}_{12}=-\nabla_{\mathbf{R}_{12}} V, \\
& \mu \ddot{\mathbf{r}}_{1}=-\nabla_{\mathbf{r}_{1}} V-\psi_{1} \mathbf{r}_{1}, \\
& \mu \ddot{\mathbf{r}_{2}}=-\nabla_{\mathbf{r}_{2}} V-\psi_{2} \mathbf{r}_{2},
\end{aligned}
$$

where the Lagrangian multipliers $\psi_{1}$ and $\psi_{2}$ are introduced in order to satisfy the constant interatomic separation constraints. ${ }^{16,22}$ In particular,

$$
\begin{aligned}
& \psi_{1}=\frac{1}{d^{2}}\left(\nabla_{\mathbf{r}_{1}} V \cdot \mathbf{r}_{1}+\mu \dot{\mathbf{r}}_{1}^{2}\right), \\
& \psi_{2}=\frac{1}{d^{2}}\left(\nabla_{\mathbf{r}_{2}} V \cdot \mathbf{r}_{2}+\mu \dot{\mathbf{r}}_{2}^{2}\right),
\end{aligned}
$$

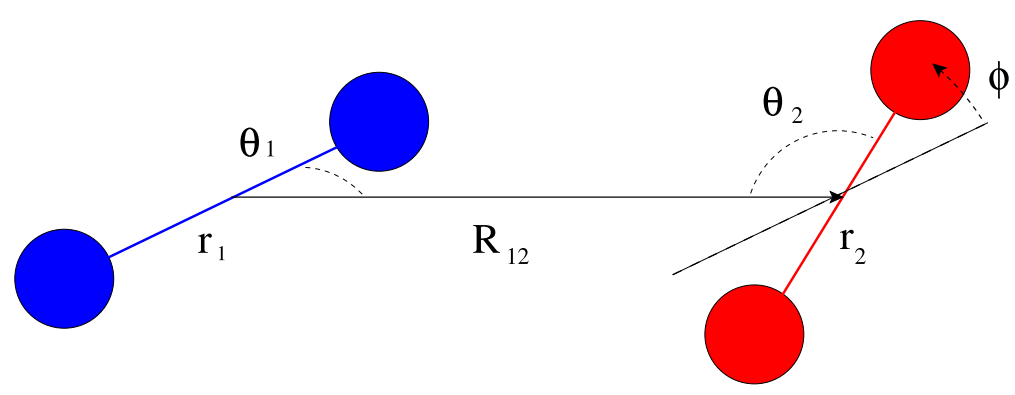

FIG. 1. Coordinate system used for the description of the PES. 
with $m_{O}$, the mass of one atom, $d$, the interatomic distance, and $\mu=m_{O} / 2$, the reduced mass. The set of 9 second-order differential equations is numerically solved using a constant-step fourth-order Runge-Kutta method. The numerical convergence of the solutions is verified by checking the conservation of the interatomic separation, the total energy, and the total angular momentum during the collision. A typical value of the time step that satisfies the above mentioned constraints with a relative error within $0.1 \%$ is $0.001 R_{m}\left(\epsilon_{m} / m_{O_{2}}\right)^{-1 / 2}$ (corresponding to $\approx 2.0 \mathrm{fs}$ ), where $m_{O_{2}}$ is the mass of $\mathrm{O}_{2}$ molecule, whereas $R_{m}$ and $\epsilon_{m}$ are a characteristic radius and interaction energy, associated with the isotropic part of $V, V^{000}\left(R_{12}\right)$, as described in Appendix A.

\section{DESCRIPTION OF CT-DSMC IMPLEMENTATIONS}

In the considered flows, intrinsic angular momenta have no preferential alignment. Hence, the dilute $O_{2}$ gas is assumed to be governed by the following Boltzmann equation:1,23

$$
\frac{\partial f}{\partial t}+\mathbf{v} \circ \frac{\partial f}{\partial \mathbf{R}}=Q(f, f) .
$$

In Eq. (8), $f(\mathbf{R}, \mathbf{v}, \epsilon, t)$ is the distribution function of the single molecule phase space states, characterized by position $\mathbf{R}$, velocity $\mathbf{v}$, and rotational energy $\epsilon$. The latter is related to the intrinsic molecular angular momentum $\boldsymbol{\Gamma}$ and moment of inertia $\mathcal{I}=m_{\mathrm{O}_{2}} \frac{d^{2}}{4}$ by the simple relationship $\epsilon=\frac{1}{2} \frac{\Gamma^{2}}{I}$. Equation (8) has been solved numerically by combining CT collisions treatment with a quite standard DSMC scheme. ${ }^{5}$ As is well known, DSMC provides a stochastic approximation of $f$ through a set of particles. In the present implementation, each particle is characterized by its center of mass position $\mathbf{R}$, velocity $\mathbf{v}$, and by its angular momentum $\boldsymbol{\Gamma}$. The time evolution of $f$, from time $t$ to time $t+\Delta t$, is computed by performing two sub-steps. In the first one, the term $Q(f, f)$ is neglected and free flight occurs. The molecule center of mass moves along a straight line with constant velocity $\mathbf{v} ; \boldsymbol{\Gamma}$ is also constant and the molecule rotates with constant angular velocity $\omega=\frac{\Gamma}{I}$. Rotation occurs in the plane normal to $\boldsymbol{\Gamma}$ (or $\omega$ ). At the end of the free flight step, particles are sorted into the cells of the spatial grid and in each cell a homogeneous relaxation occurs, which changes particles velocity $\mathbf{v}$ and angular momentum $\boldsymbol{\Gamma}$ through binary collisions. The number of collisions to be computed in each cell is estimated by a majorant collision frequency scheme. ${ }^{5}$ An upper bound $\bar{N}_{c o l l}$ for the collision number in a cell of volume $V_{c}$, containing $N_{p}$ particles, is obtained as

$$
\bar{N}_{\text {coll }}=\frac{1}{2} \frac{N_{p}}{V_{c}} N_{p} \pi b_{\max }^{2} g_{\max } \Delta t,
$$

where $g_{\max }$ is an upper bound for the modulus of relative molecular velocities and $b_{\max }$ is the maximum value of the impact parameter $b$ beyond which no molecular interaction occurs. In the present work, $b_{\max }$ has been set equal to the cutoff radius $4 R_{m}$, thus ensuring that significant collisions are taken into account. Acceptance-rejection is used by generating a sequence of $\bar{N}_{\text {coll }}$ randomly selected molecule pairs. A given pair $(i, j)$ is accepted for a collision whenever a random fraction $r_{f}$ is less than the ratio $\frac{\left\|\mathbf{v}_{j}-\mathbf{v}_{i}\right\|}{g_{\max }}$. For each collision, a classical trajectory calculation is performed. Initial relative position $R_{12}$ is constructed by placing the center of mass of molecule " 1 " in the center of a sphere of radius $4 R_{m}$ and molecule " 2 " on the surface of the sphere, having sampled the impact parameter $b$ from the linear probability density function (p.d.f.) $\mathcal{P}(b)=\frac{2}{b_{\max }^{2}} b$, with $0<b<b_{\max }$. The initial values of $\mathbf{r}_{1}$ and $\mathbf{r}_{2}$ and their time derivatives are computed from respective molecular angular momentum vectors $\boldsymbol{\Gamma}_{1}$ and $\boldsymbol{\Gamma}_{2}$ which are conserved during the free flight step of the DSMC scheme. In particular, for each molecule, the direction of the molecular axis $\mathbf{r}_{i}$ is randomly chosen in the plane perpendicular to the direction of the angular momentum.

Collisions occurring in the whole computational domain in a time step $\Delta t$ can be divided into groups to be concurrently computed by a multiprocessor machine. The beneficial effects of GPU acceleration on CT-DSMC simulations has been shown in Refs. 18 and 19. In this work, two different parallel architectures and parallelization strategies have been used. The first, based on OpenMP programming environment, has been adopted to run CT-DSMC simulations on a workstation equipped with two six-cores processors. In this particular implementation, an otherwise 
sequential CT-DSMC code uses fork-join OpenMP directives to distribute groups of collisions among a number of concurrent threads. A second strategy, combining MPI and OpenMP, has been adopted to run a number of statistically independent simulations of (macroscopically) identical systems on the nodes of the CINECA IBM Blue Gene/Q. Depending on the discretization parameters, the heat transfer simulations presented in Sec. V A 2 require approximatively from 11 to $16 \mathrm{~h}$ on Blue Gene/Q with a time required to process a collision comprised between 6.1 and $7.6 \mu$ s. It should be observed that the concurrent calculation of collisions dynamics might give rise to problems when the same particle appears more than once as a collision partner in the list of accepted collision pairs in a given cell. In this case, more than one post-collision state is computed for the same particle. In the sequential version of the algorithm, the problem does not show up, since collisions in a given cell are executed in time ordered sequence. If a particle is selected more than once, it simply suffers a series of collisions, each of them changing its state. As is obvious, the problem can be minimized by reducing the time step (and $\bar{N}_{c o l l}$ ) or increasing $N_{p}$. However, it cannot be fully eliminated and it has been dealt with by considering collisions involving the same particle as time ordered events which attribute to the particle the state resulting from the last collision.

\section{IV. $\mathrm{O}_{2}$ TRANSPORT PROPERTIES' ESTIMATIONS}

As anticipated in the Introduction, $O_{2}$ transport properties have been obtained by different methods, all of them based on the CT calculations described above. The adopted methods can be divided into two groups. The first group contains two well known techniques, specifically designed for transport properties' calculations. The first of them (CT-MC), briefly described in Sec. IV A, combines CT calculations with Monte Carlo sampling from an equilibrium state to directly evaluate transport properties from generalized Chapman-Enskog expansions ${ }^{10,11}$ for a gas of linear rigid rotators. ${ }^{10,12}$ The second technique, described in Sec. IV B, exploits Green-Kubo (GK) formalism ${ }^{24}$ to obtain transport properties from the fluctuations spectra in an equilibrium unconfined gas. The simple tagged particles diffusion (TPD), used to evaluate $\mathrm{O}_{2}$ self-diffusion coefficient and described in Sec. V, can also be included into the first group of methods, being based on equilibrium simulations. The second group of techniques consists in non-equilibrium CT-DSMC simulations of $\mathrm{O}_{2}$ flows, whose setup has been designed to obtain a specific transport property. The first of them, described in Sec. V A 1, aims at evaluating shear viscosity from CT-DSMC simulations of the uniform shear flow (USF), whereas the second one, described in Sec. V A 2, obtains the thermal conductivity from the heat flux and temperature profiles in $\mathrm{O}_{2}$ confined between two parallel solid plates kept at different temperatures.

\section{A. Direct evaluation of transport properties from Chapman-Enskog expansions}

Expressions for the transport coefficients of a diatomic gas composed of classical rigid rotators have been derived by Taxman in $1958 .{ }^{10}$ Their explicit forms, corresponding to the first approximation of the classical Chapman-Enskog theory, are given in Appendix B for sake of completeness. As in the case of a monatomic gas, the evaluation of transport coefficients involves the calculations of multiple integrals in which the gas equilibrium distribution function plays the role of a probability density multiplying functions of post-collision states. Hence, the evaluation of transport coefficients is performed by a straightforward Monte Carlo quadrature method ${ }^{12,14}$ in which CT provides post-collision states from pre-collision initial states sampled by the proper equilibrium distribution. In the particular implementation of the CT-MC method adopted here, the evaluation of each transport coefficient at a given temperature has been based on averaging 24 statistically independent samples, each composed of $4 \times 10^{5}$ classical trajectories. The transport coefficients standard error, estimated from the variance of the 24 sample distributions, amounts to $2.4 \%-2.7 \%$ for shear viscosity, to $4.8 \%-7.1 \%$ for bulk viscosity, and to $2.6 \%-3.1 \%$ for thermal conductivity, in the temperature range 300-900 K. Hence, the standard errors associated with averaging over 24 samples are estimated to be about five times smaller, i.e, well below $1 \%$ for shear viscosity and thermal conductivity and around $1 \%$ for bulk viscosity. CT-MC evaluation of transport coefficient, 
as expected, is the most efficient among the methods considered here. Producing the 24 samples described above takes $20-30 \mathrm{~min}$ on a 12 cores workstation. However, the method is based on the availability of explicit expressions for transport coefficients, whereas the methods/techniques described below, although less efficient, do not need underlying Chapman-Enskog expansions.

\section{B. Green-Kubo expressions for the transport coefficients}

Linear response theory is a powerful tool for the description of the relaxation towards thermodynamic equilibrium of any system subject to small perturbations. The fluctuation-dissipation theorem, in particular, connects the time correlation functions of mechanical quantities to the system transport properties. These relations are known as the Green-Kubo expressions for the transport coefficients. ${ }^{24}$ This theory being independent of the mechanical model describing the system, it has been primarily applied to the evaluation of transport properties by equilibrium molecular dynamics simulations of liquids ${ }^{25}$ and solids (see, e.g., Ref. 26 and references therein). In this study, we apply the Green-Kubo formulas to the evaluation of transport coefficients in CT-DSMC simulations of dilute (i.e., ideal) gases. It has been shown ${ }^{27}$ that the fluctuations in DSMC simulations exhibit the correct physical behaviour. The standard approach usually applied to molecular dynamics simulations ${ }^{20,25,26}$ can therefore be applied to CT-DSMC simulations. The reader is referred to the relevant literature for details. Here, we briefly recall the main results. For a system of volume $V_{o l}$ in equilibrium at temperature $T$ and pressure $p$, the Green-Kubo formulas read ${ }^{20}$

$$
\begin{gathered}
\eta=\frac{1}{V_{o l}} \frac{1}{k_{\mathrm{B}} T} \int_{0}^{\infty}\left\langle J_{x y}^{p}(0) J_{x y}^{p}(\tau)\right\rangle d \tau, \quad \lambda=\frac{1}{V_{o l}} \frac{1}{k_{\mathrm{B}} T^{2}} \int_{0}^{\infty}\left\langle J_{x}^{q}(0) J_{x}^{q}(\tau)\right\rangle d \tau, \\
\frac{4}{3} \eta+\kappa=\frac{1}{V_{o l}} \frac{1}{k_{\mathrm{B}} T} \int_{0}^{\infty}\left\langle J_{x x}^{p}(0) J_{x x}^{p}(\tau)\right\rangle d \tau,
\end{gathered}
$$

where $\eta$ is the shear viscosity, $\kappa$ the bulk viscosity, and $\lambda$ the thermal conductivity. The brackets $\langle\cdots\rangle$ denote the canonical ensemble average.

The quantities $J$, the currents, are spatially-averaged, time-dependent mechanical quantities described below. For an ideal gas system, shear and bulk viscosities are evaluated by the following current:

$$
\mathbf{J}^{p}=\sum_{i} m \mathbf{c}_{i} \mathbf{c}_{i}-p V_{o l} \mathbf{I},
$$

where the sum runs over gas molecules of mass $m$ and peculiar velocity $\mathbf{c}_{i}$. This is a symmetric tensor of rank two whose off-diagonal elements appear in the Green-Kubo formula for shear viscosity, Eq. (10), whereas the diagonal terms appear in Eq. (11). Analogously, the thermal conductivities are obtained from the vector current,

$$
\mathbf{J}^{q}=\sum_{i} E_{i} \mathbf{c}_{i}
$$

where $E_{i}$ is molecular energy. Setting

$$
E_{i}=\frac{1}{2} m_{i} c_{i}^{2}
$$

provides the translational thermal conductivity, whereas setting

$$
E_{i}=\frac{1}{2} m_{i} c_{i}^{2}+\epsilon_{i}
$$

provides the total thermal conductivity. In Eq. (15), $\epsilon_{i}$ is the molecule internal energy. Note that three independent components of the current are available for the calculation of each transport coefficient. The integrals in Eqs. (10) and (11) are calculated as the zero-frequency limit of the current power spectrum. In a CT-DSMC simulation, the currents are evaluated by summing over all simulated particles. For each transport coefficient $v$, we sample the related current $J^{v}$ at $2 N_{t}$ discrete time points,

$$
J_{i}^{v}=J^{v}\left(t=t_{i}\right), \quad i=0, \ldots, 2 N_{t}-1,
$$


we then apply standard Fast Fourier Transform (FFT) algorithms ${ }^{28}$ to get the current Fourier transform,

$$
H_{n}^{v}=H^{v}\left(f=f_{n}\right), \quad n=-N_{t}, \ldots, N_{t} .
$$

Discrete frequencies are related to the sampling times via

$$
\begin{aligned}
& t_{i}=i * \Delta t, \quad i=0, \ldots, 2 N_{t}-1, \\
& f_{n}=\frac{n}{2 N_{t} \Delta t}, \quad n=-N_{t}, \ldots, N_{t} .
\end{aligned}
$$

Next, the power spectrum is computed as

$$
P_{n}^{v}=\left|H_{n}^{v}\right|^{2}, \quad n=0, \ldots, N_{t} .
$$

The transport coefficients are obtained from

$$
\begin{gathered}
\eta=\frac{1}{V_{o l}} \frac{1}{k_{\mathrm{B}} T} \frac{1}{2 t_{\text {sim }}} P_{0}^{\eta} \cdot w, \\
\frac{4}{3} \eta+\kappa=\frac{1}{V_{o l}} \frac{1}{k_{\mathrm{B}} T} \frac{1}{2 t_{\text {sim }}} P_{0}^{\eta_{v}} \cdot w, \\
\lambda=\frac{1}{V_{o l}} \frac{1}{k_{\mathrm{B}} T^{2}} \frac{1}{2 t_{\text {sim }}} P_{0}^{\lambda} \cdot w,
\end{gathered}
$$

$t_{\text {sim }}$ being the simulation duration and $w$ being the weight of simulated particles (each particle representing $w$ real particles). The factor $w$ arises because the fluctuation amplitude is proportional to the square root of the number of simulated particles. For each temperature, the calculation proceeds as follows. A 0-dimensional, microcanonical simulation is set up fixing the number of simulated particles, the simulation volume, and the total energy. The number of simulated particles is 1000 . The sampling time interval is set equal to the simulation time step. The simulation duration should be large when compared with the characteristic relaxation times. In this case, the largest relevant relaxation time is the rotational relaxation time. For this work, simulation times of $t_{\text {sim }} / \tau_{c}=70-87$ and time steps of $d t_{\text {sim }} / \tau_{c}=0.0021-0.0026$ are used. Here, $\tau_{c}$ is the mean collision time defined as the ratio between the mean free path, $l$, based on viscosity, and the reference velocity $\sqrt{R T}$, $\tau_{c}=l / \sqrt{R T}$. During the simulation, time series of the relevant currents are recorded. At the end of the simulation, the time series are Fourier transformed and squared to get the currents' power spectra, Eq. (20); these are proportional to frequency-dependent extensions of the transport coefficients. ${ }^{20}$ Transport coefficients are then obtained from Eqs. (21)-(23). This is illustrated in Fig. 2 where the frequency-dependent thermal conductivity obtained from a CT-DSMC simulation at $T=300 \mathrm{~K}$ is plotted. The classical transport coefficient is obtained as the zero-frequency limit; it is also apparent how the correlation drops quickly as the collisionless limit is approached $\left(\omega \tau_{c}>1\right)$. Finally, results are ensemble averaged over a number of independent runs. For this work, results have been obtained averaging over 1000 independent runs; as a result, statistical scatter on measured transport coefficients is below $3 \%$. It is worth noting that a single run yields all the transport coefficients at the same time.

\section{SELF-DIFFUSION}

The self-diffusion coefficient has been computed in the framework of the CT-DSMC using the tagged particle diffusion method. ${ }^{29,30}$ This method is based on the computation of the variance of the net displacement of the simulated particles in an equilibrium condition. The time derivative of the variance of the net displacement of the particles $\left\langle r^{2}\right\rangle$ is constant and is related to the self-diffusion coefficient by the following equation: ${ }^{29,30}$

$$
\mathcal{D}=\frac{1}{6} \frac{d\left\langle r^{2}\right\rangle}{d t} .
$$

In the present work, the self-diffusion coefficient has been computed at atmospheric pressure and at different temperatures using 10000 particles for each simulation. Numerically, the time derivative 


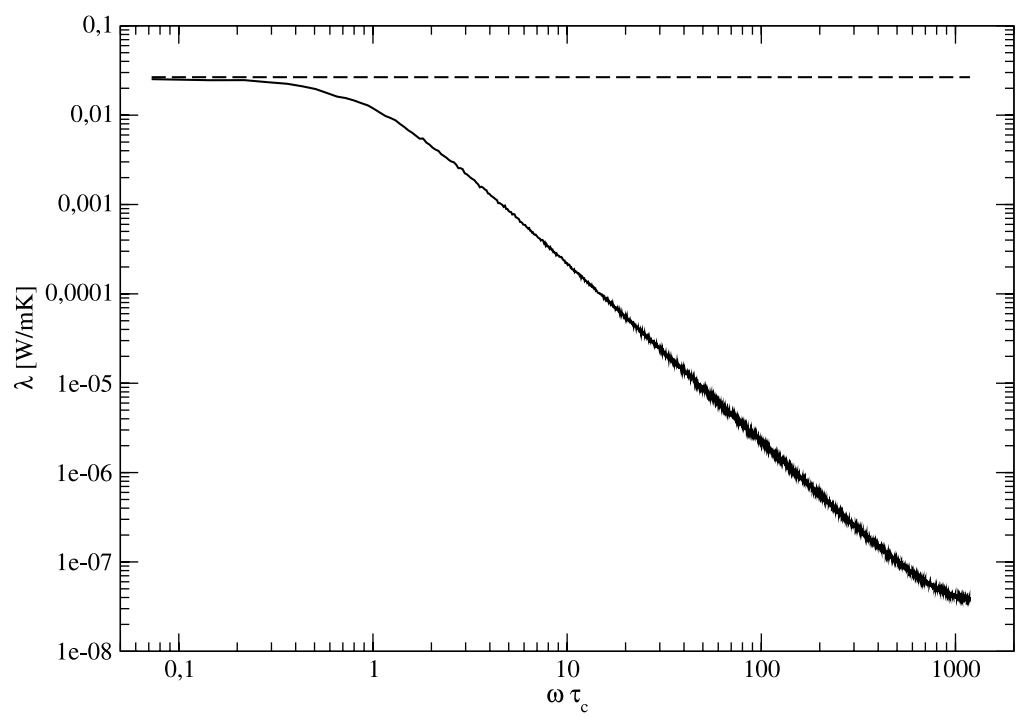

FIG. 2. Frequency dependent thermal conductivity versus dimensionless frequency at $300 \mathrm{~K}$. Solid line: numerical results and dashed line: extrapolation to zero frequency.

in Eq. (24) has been obtained from the slope of the straight line that fits in the least square sense the time evolution of $\left\langle r^{2}\right\rangle$. The results are reported in Fig. 3. Reference data have been taken from Ref. 31 where recommended collision integrals are the universal function fits from Ref. 32. These data have a quoted accuracy of $20 \%$. As can be seen from Fig. 3, the results are in close agreement with the reference data at all the investigated temperature values.

\section{A. Transport properties evaluation from non-equilibrium CT-DSMC simulations}

$\mathrm{O}_{2}$ shear viscosity $\eta$ and thermal conductivity $\lambda$ have also been computed from two distinct flow setups. The first one, designed to compute $\eta$, is based on the stress tensor calculation in a gas subject to uniform shear, whereas $\lambda$ is estimated from the steady heat flux in a gas confined between two infinite parallel plates kept at different temperatures.

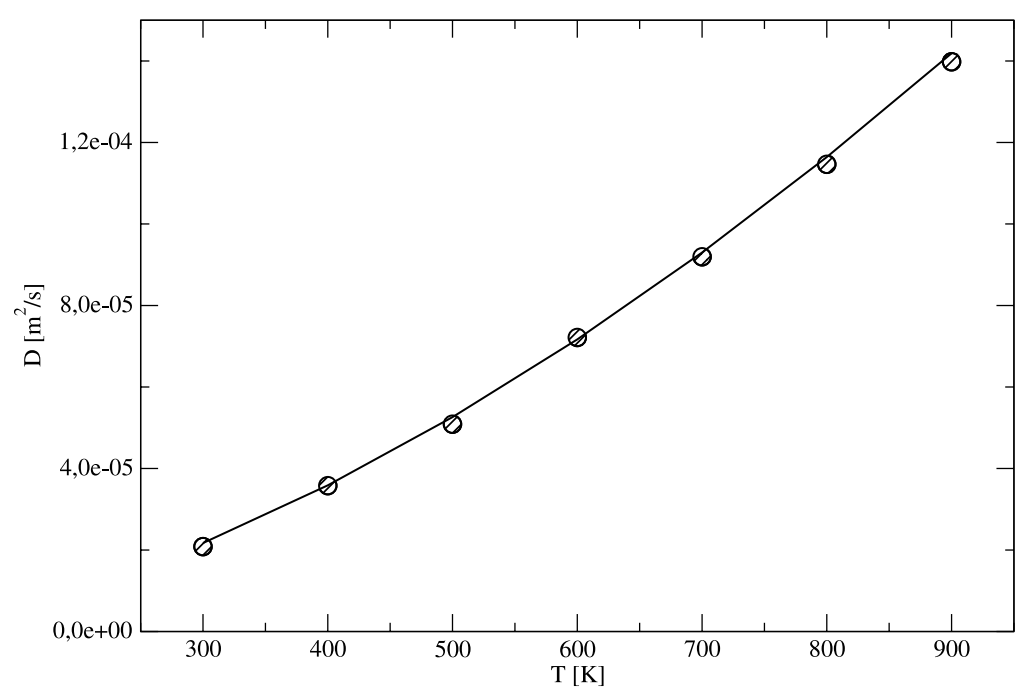

FIG. 3. Diffusion coefficient at atmospheric pressure. Shaded circles: present work and solid line: from Ref. 31. 


\section{Uniform shear flow in a diatomic gas}

USFs have been extensively studied ${ }^{33-35}$ by kinetic equations, in order to obtain a description of rheology of a fluid arbitrarily far from equilibrium. Analytical and numerical USF investigations, ranging from monatomic gases ${ }^{33}$ to dilute and dense granular media, ${ }^{34,35}$ have shown that, as expected, the linear relationship between shear stress and shear rate, at the basis of Navier-Stokes constitutive law, is obtained as the small shear rate limit of the kinetic shear stress. ${ }^{33}$ Therefore, $O_{2}$ shear viscosity can be obtained by the calculation of the shear stress in a flow configuration in which the gas fills the whole physical space with uniform and constant densities. The gas is subject to uniform shear. Hence, the bulk velocity field $\mathbf{u}$ is assigned as

$$
u_{x}=0, \quad u_{y}=\alpha x, \quad u_{z}=0
$$

by a suitable choice of the reference frame. In Eq. (25), $\alpha$ is the imposed uniform shear rate. Since spatial gradients have only a non-zero component along $x$, the problem can be studied by the spatially one-dimensional Boltzmann equation,

$$
\frac{\partial f}{\partial t}+v_{x} \frac{\partial f}{\partial x}=Q(f, f) .
$$

It should be now observed that the flowfield defined by Eq. (25) keeps the very same structure in any local reference frame which uses the axes defined above and whose origin moves along any stream line with the constant fluid speed. Moreover, the distribution function of molecular velocities relative to any such local reference frame does not depend on the spatial position of its origin. Hence, following previous studies, ${ }^{33-36}$ the spatial coordinate can be eliminated by seeking solutions of Eq. (26) which are invariant upon a translation of the origin of the reference system. Accordingly, $f$ is assumed to have the form

$$
f(x, \mathbf{v}, \epsilon, t)=f(\mathbf{v}-\alpha x \hat{\mathbf{y}}, \epsilon, t) .
$$

Substituting the above ansatz into Eq. (26) leads to the following problem formulation, in which the shear rate acts as a velocity dependent force on a spatially homogeneous gas:

$$
\frac{\partial f}{\partial t}-\alpha c_{x} \frac{\partial f}{\partial c_{y}}=Q(f, f)
$$

being $\mathbf{c}=\mathbf{v}-\alpha x \hat{\mathbf{y}}$, the peculiar velocity. The new problem formulation, provided by Eq. (28), not only simplifies its analytical treatment ${ }^{35}$ but also allows running space homogeneous CT-DSMC simulations in which a large number of simulation particles can be allocated to the single required cell to obtain accurate solutions.

It is to be noted that the presence of shear produces uniform and continuous heating of the gas. Gas temperatures can be kept constant by a simple Gaussian thermostat. ${ }^{22}$ In the present study, thermostatting is obtained by rescaling molecular centers of mass velocities at each time step. Then, the thermostat is equivalent to the addition of a force term $-\beta \frac{\partial}{\partial \mathbf{c}}(\mathbf{c} f)$ on the lhs of Eq. (28), which takes the form ${ }^{35}$

$$
\frac{\partial f}{\partial t}-\alpha c_{x} \frac{\partial f}{\partial c_{y}}-\beta \frac{\partial}{\partial \mathbf{c}}(\mathbf{c} f)=Q(f, f) .
$$

The value of $\beta$ is determined by the requirement that the heat dissipated by shear is carried away by the thermostat. It is to be noted that the adopted thermostat only acts on translational energy by keeping the translational temperature $T_{t}$ constant. This choice has been taken advisedly, in order to allow excessive deviations from the hydrodynamic limit to be detected through the difference between the translational and rotational temperatures. Actually, when applied to polyatomic gases, uniform shear causes a separation of the rotational temperature $T_{r}$ from the translational one, $T_{t}$. The separation extent is a growing function of $\alpha$. The effect has been preliminarily studied by computationally inexpensive DSMC simulations in which the collision term of Eq. (29) has been computed on the basis of Borgnakke-Larsen model. ${ }^{6}$ Hard sphere collision cross section has been assumed and the fraction $z$ of inelastic collisions has been set equal to 0.2. Figure 4(a) shows the time evolution of the normalized values of $T(t), T_{r}(t)$ and shear stress $\tau_{x y}(t)$ towards their respective 

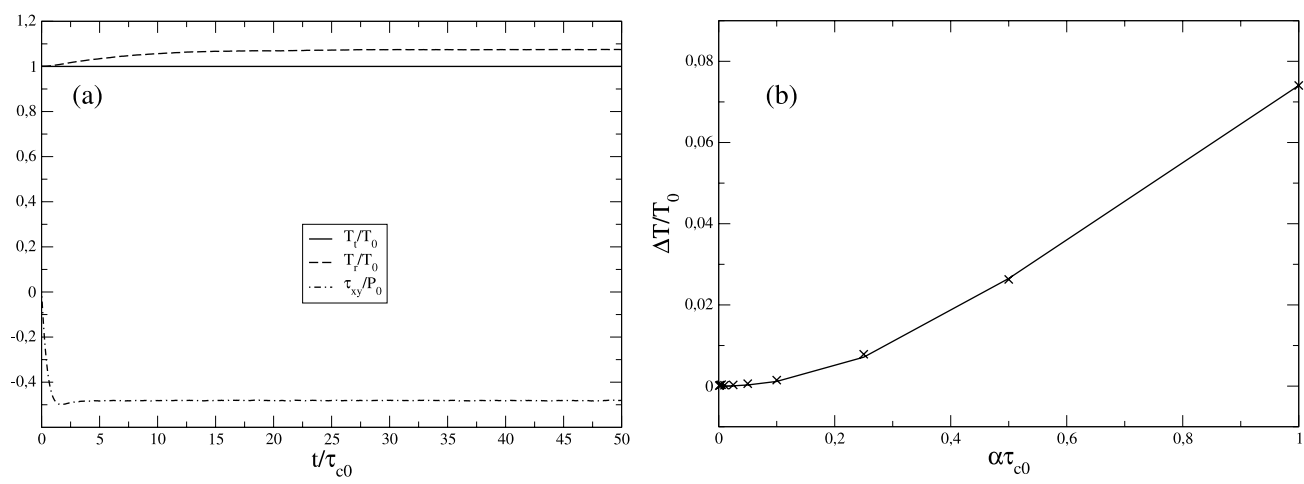

FIG. 4. DSMC simulations of USF in a diatomic gas by Borgnakke-Larsen model. ${ }^{6}$ Hard sphere collision cross section, $z=0.2$; number of simulation particles $N_{p}=10^{6}$. Left-Time evolution of $T / T_{0}, T_{r} / T_{0}$ and $\tau_{x y} /\left(n_{0} k_{\mathrm{B}} T_{0}\right), \alpha \tau_{c 0}=1.0$. Right $-\Delta T / T_{0}$ as a function of $\alpha \tau_{c 0} . \tau_{c 0}=l_{0} / \sqrt{R T_{0}}$ is the reference mean free time, $l_{0}=1 /\left(\sqrt{2} \pi n_{0} \sigma_{h s}^{2}\right)$ is the reference mean free path in a gas of hard spheres of diameter $\sigma_{h s}$.

constant limit values, when the normalized shear rate $\alpha$ is equal to one. In the initial equilibrium state, $T(0)$ and $T_{r}(0)$ are equal. The shear driven translational non-equilibrium is gradually transferred to rotational degrees of freedom till collisional energy exchange between translational and rotational motions prevents further growth of $T_{r}$. The normalized separation $\frac{\Delta T}{T_{0}}=\lim _{t \rightarrow+\infty} \frac{T_{r}-T_{0}}{T_{0}}$, shown in Figure 4(b), is an even function of the shear rate, which vanishes more rapidly than $\alpha$.

Hence, in the limit of small shear rates, thermostatting molecular velocities is sufficient to keep $T_{r}$ very close to the reference temperature $T_{0}$.

In the present study, Eq. (29) has been solved numerically by the CT-DSMC scheme outlined in Sec. III, replacing the free flight step in the physical space with a sub-step changing molecular centers of mass velocities as if they were subject to a force $\mathbf{F}_{s}=-\alpha c_{x} \hat{\mathbf{y}}$. The effect of the thermostatic force has been taken into account by rescaling centers of mass velocities at each time step in order to $\operatorname{keep} T(t)=T_{0}$.

The viscosity of the gas has then been obtained as $\lim _{\alpha \rightarrow 0} \frac{\tau_{x y}}{\alpha}$, where $\tau_{x y}=m_{O_{2}} \int c_{x} c_{y} f d \mathbf{c} d \epsilon$ is $x y$ component of the stress tensor, computed from CT-DSMC simulations. The function $\tau_{x y}(\alpha) / \alpha$ has been evaluated at a discrete set of shear rate values and fitted to the polynomial,

$$
\frac{\tau_{x y}}{\alpha}=\eta_{0}+\eta_{2} \alpha^{2}+\eta_{4} \alpha^{4}
$$

since the ratio $\tau_{x y} / \alpha$ must be an even function. In the limit of vanishing $\alpha, \tau_{x y}$ is proportional to the imposed shear through the shear viscosity coefficient. Hence, the viscosity corresponds to the coefficient $\eta_{0}$ in Eq. (30). This process has been repeated for each temperature of interest. Figure 5 shows the obtained values of the ratio $\tau_{x y} / \alpha$ and their corresponding interpolation at two different temperatures, $400 \mathrm{~K}$ and $900 \mathrm{~K}$. Depending on the value of the shear rate $\alpha$, typical simulations have been done using 40000-100000 molecules with a dimensionless time step $\Delta t / \tau_{c} \simeq 0.0025$. In order to assess the statistical uncertainty of the results, 10 independent simulations have been performed for each shear rate. The obtained viscosities are compared with those computed from CT-MC method based on Taxman expressions ${ }^{10}$ and Green-Kubo ${ }^{24}$ expressions from equilibrium fluctuations spectra (GK). Figure 6 shows the results obtained at different temperatures between $300 \mathrm{~K}$ and $900 \mathrm{~K}$. The statistical uncertainty of the obtained results for all the three methods is within 3\%. As it can be seen, all the three methods are in close agreement with each other at all considered temperatures. Moreover the results are in close agreement with the reference data of Lemmon and Jacobsen. ${ }^{37}$

\section{Thermal conductivity estimation}

As for the shear viscosity coefficient, $O_{2}$ thermal conductivity has been also estimated by non-equilibrium CT-DSMC simulations. The spatially one dimensional form of Eq. (8) has been 

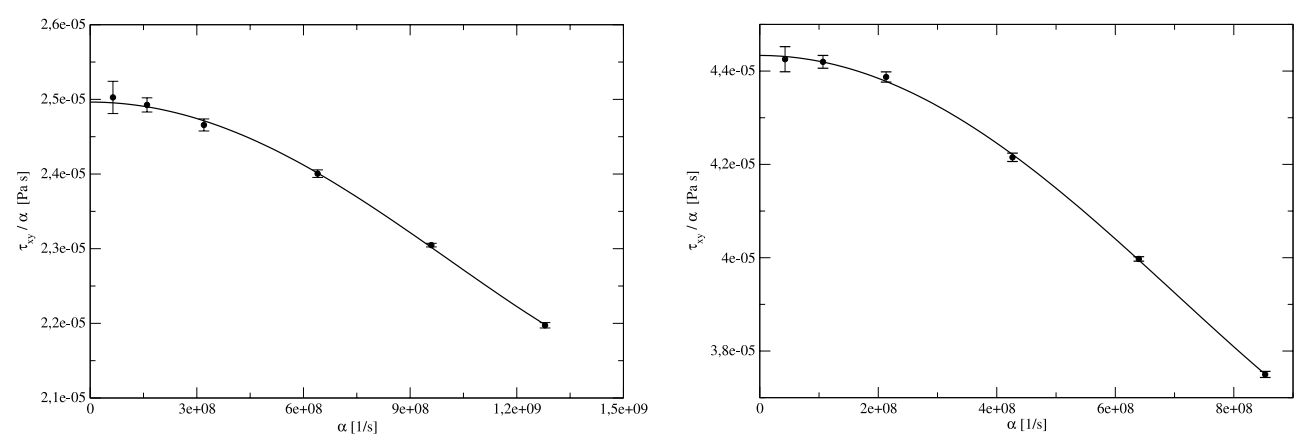

FIG. 5. Ratio $\tau_{x y} / \alpha$ at different shear velocities and temperatures. Filled circles: numerical simulations obtained with the CT-DSMC method, solid line: polynomial fit of CT-DSMC data based on Eq. (30). Left panel: $400 \mathrm{~K}$ and right panel: $900 \mathrm{~K}$.

adopted to study the classical problem of the steady heat flux in a dilute gas confined in the gap between two infinite parallel plates kept at uniform and constant temperatures $T_{c}$ and $T_{h}\left(T_{c} \leq T_{h}\right)$, respectively. Gas-wall interaction has been treated assuming full accommodation for both translational and rotational energies. The gas fills the gap with average number density $n_{0}=\frac{1}{L} \int_{0}^{L} n(x) d x$. For this problem, a global Knudsen number $K n$ can be defined as

$$
K n=\frac{16}{5 \sqrt{2 \pi}} \frac{\eta_{0}}{P_{0}} \sqrt{\frac{k_{\mathrm{B}} T_{0}}{m_{O_{2}}}} \frac{1}{L},
$$

where $L$ is the gap width, $P_{0}=n_{0} k_{\mathrm{B}} T_{0}, T_{0}=\left(T_{c}+T_{h}\right) / 2$, and $\eta_{0}=\eta\left(T_{0}\right)$ are the reference pressure, temperature, and viscosity, respectively. A $K n$ dependent effective thermal conductivity $\lambda_{\text {eff }}(T, K n)$ can be formally defined from the relationship

$$
q_{x}=-\lambda_{e f f}(T, K n) \frac{d T}{d x}
$$

where $x$ is the spatial coordinate, $q_{x}=\int c_{x}\left(\frac{m}{2} \mathbf{c}^{2}+\epsilon\right) f(\mathbf{c}, \epsilon) d \mathbf{c} d \epsilon$ is the constant heat flux component normal to the plates, and $T=\left(3 T_{t}+2 T_{r}\right) / 5$ is the overall gas temperature. Extensive analytical and numerical investigations ${ }^{38-40}$ have shown that, when $K n$ is sufficiently small, $\lambda_{\text {eff }}$ is close to the Chapman-Enskog thermal conductivity $\lambda(T)$ in a central region of the gap but, as expected, it deviates from $\lambda(T)$ in the vicinity of the walls. This central strip is isolated from the two Knudsen layers next to the plates and it is not affected by the wall temperature jumps (see right panel of Figure 7), whose presence only sets the slop of temperature profile. It is sufficient that $K n$ is close to 0.025 to obtain that the relative error $\left|\lambda_{\text {eff }}-\lambda\right| / \lambda$ does not exceed $10^{-2}$ in the center of the gap. ${ }^{39}$ Following the flow and computational settings given in Refs. 39 and $40, \lambda\left(T_{0}\right)$ has been
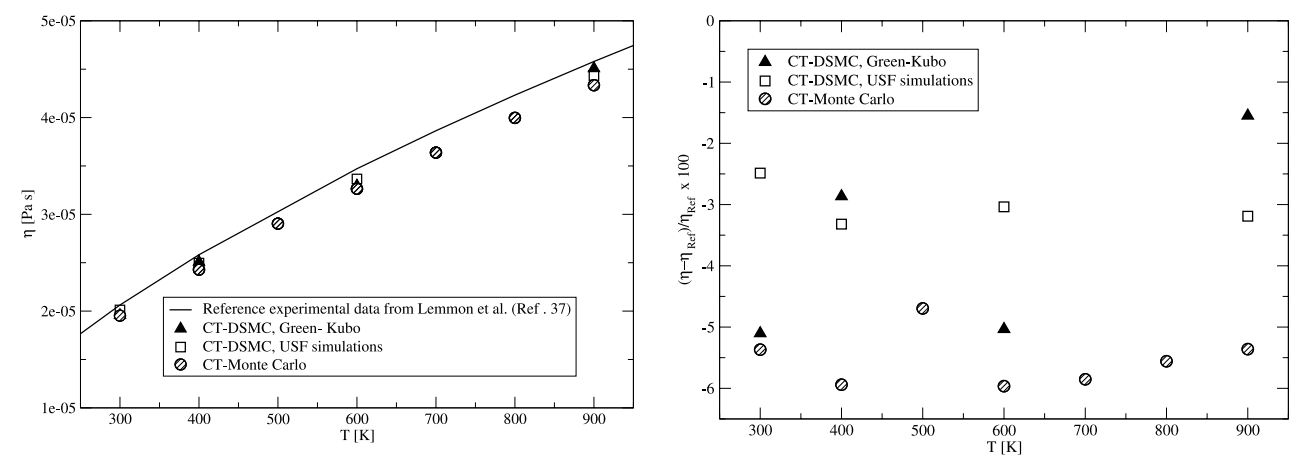

FIG. 6. Left-Temperature dependence of $\boldsymbol{O}_{2}$ shear viscosity estimation by different methods and comparison with reference experimental data. ${ }^{37}$ Right-Relative deviations from reference data. 

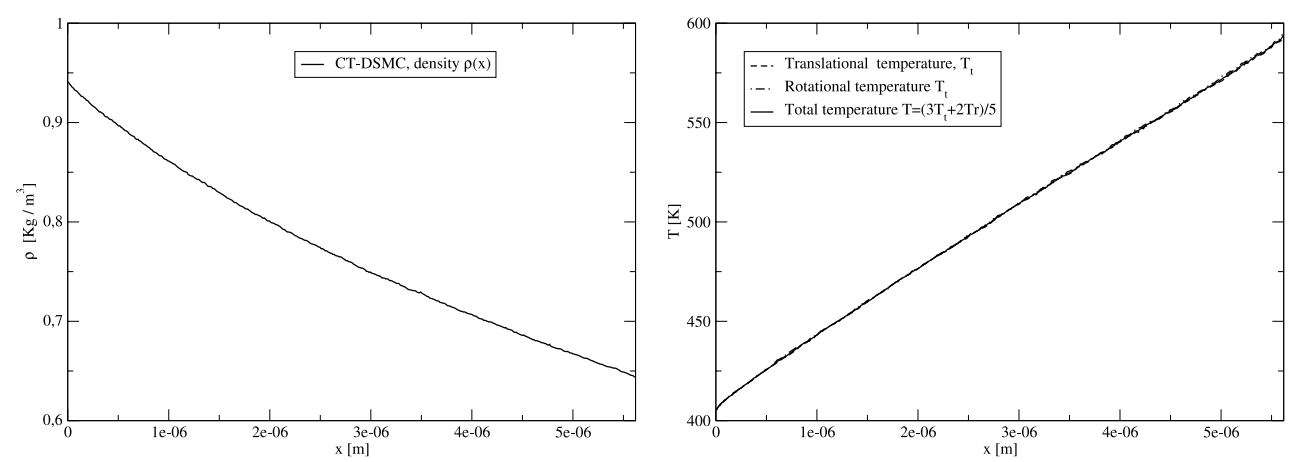

FIG. 7. Density and temperatures profiles in heat flux between parallel plates. Hot wall temperature $T_{h}=600 \mathrm{~K}$ and cold wall temperature $T_{c}=400 \mathrm{~K}$. Reference Knudsen number $K n \simeq 1 / 36$, based on $O_{2}$ viscosity at $\left(T_{h}+T_{c}\right) / 2$.

estimated from Eq. (32). Heat flux and temperature profiles have been obtained by carefully designed CT-DSMC simulations of heat conduction. The thermal conductivity estimates have been computed from ensemble averages of 2048 macroscopically identical CT-DSMC runs. The temperature gradient, $\left(\frac{d T}{d x}\right)_{x=x_{0}}$, has been computed at a spatial position $x=x_{0}$, next to center of the gap, where the temperature takes the value $T_{0}$. The temperature gradient has been computed by a linear fit of the temperature profile in a narrow interval centered on $x_{0}$. For each individual CT-DSMC simulation, the temperature ratio between the hot and cold walls $T_{h} / T_{c}$ has been set equal to 1.5 , the Knudsen number $K n$, based on the viscosity, has been set equal to $\simeq 1 / 36$. The chosen flow setting also ensures that the translational and rotational temperatures are very close, as shown in the right panel of Figure 7. The uniform grid size $\Delta x$ and time step $\Delta t$ have been, respectively, set equal to 0.08 reference mean free paths and 0.004 reference mean free times. Not less than 270 particles per mean free path have been used. To reduce the duration of the transient, each CT-DSMC simulation has been started from the particles states sampled from a companion steady DSMC simulation using the same computational settings but based on hard sphere collision cross section and Borgnakke-Larsen model for internal degrees of freedom. As an example, Figure 7 shows the computed density and temperature profiles when the hot wall temperature is $T_{h}=600 \mathrm{~K}$ and the cold wall temperature is $T_{c}=400 \mathrm{~K}$.

The computed thermal conductivities are compared with those obtained using the methods of Taxman ${ }^{10}$ and Green-Kubo. ${ }^{24}$ Figure 8 shows the computed results at different temperatures between $300 \mathrm{~K}$ and $900 \mathrm{~K}$. The three methods are in good agreement between each other but, when compared with the reference results of Lemmon and Jacobsen ${ }^{37}$ they show an error increasing with temperature. Although the discrepancies are of the same order of the accuracy of the reference results, the underestimation of the thermal conductivity is apparent and some considerations are needed in order to justify this behavior.
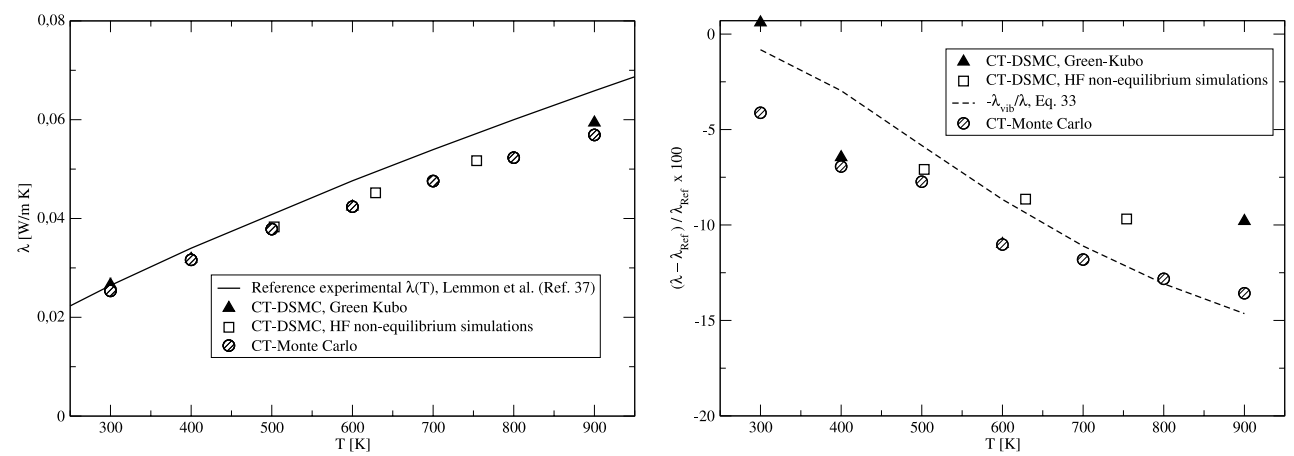

FIG. 8. Left-Temperature dependence of $\mathrm{O}_{2}$ thermal conductivity estimations by different methods and comparison with reference experimental data ${ }^{37}$ Right-Relative deviations from reference data. 
As mentioned in the Introduction, in the present work, only the rotational degrees of freedom have been considered whereas the other internal degrees of freedom have been disregarded. Although this assumption has not a significant impact in the viscosity estimation, the same conclusion does not hold for the thermal conductivity. Following the work of Uribe et al. ${ }^{41}$ the different contributions of the internal degrees of freedom to the thermal conductivity can be quantified in a simplified formulation. In particular, between $300 \mathrm{~K}$ and $900 \mathrm{~K}$, the vibrational degrees of freedom represent the most significant contribution to thermal conductivity after those related to the translational and rotational energies. This contribution can be quantified in the following way: ${ }^{41}$

$$
\lambda_{\text {vib }}=\rho \mathcal{D}_{\text {vib }} c_{\text {vib }}
$$

where $\rho$ is the gas density, $\mathcal{D}_{\text {vib }}$ is the diffusion coefficient of the vibrational energy, and $c_{v i b}$ is the specific heat of the vibrational degrees of freedom.

Equation (33) is based on the hypothesis that the vibrational energy is only transported by particles and can be characterized by a proper diffusion coefficient. Due to the difficulties in obtaining $\mathcal{D}_{v i b}$, its value is usually approximated by the self-diffusion coefficient. On the right panel of Fig. 8, the fraction $\lambda_{v i b} / \lambda$ (dashed line) is compared with the discrepancies obtained in the present work. Both data show similar behavior and they are quantitatively of the same order of magnitude. This confirms that the underestimation in the calculated thermal conductivities with respect to the recommended values is, to a large extent, ascribable to the neglect of the vibrational modes.

\section{BULK VISCOSITY}

Bulk viscosity has been computed only by the CT-MC technique, according to Eq. (B2). Its value could also be obtained from Green-Kubo formulation but the low signal to statistical noise ratio associated with the CT-DSMC implementation described in Sec. IV B has prevented reaching satisfactory accuracy. The results are shown in Fig. 9 as circles. Since there is considerable scatter in the literature values for this coefficient, it is interesting to compare the present results with the literature results from different sources.

Most experimental determinations of the bulk viscosity, ${ }^{42-46}$ use ultrasound absorption measurements since rotational relaxation produces additional sound absorption with respect to the structureless gas.

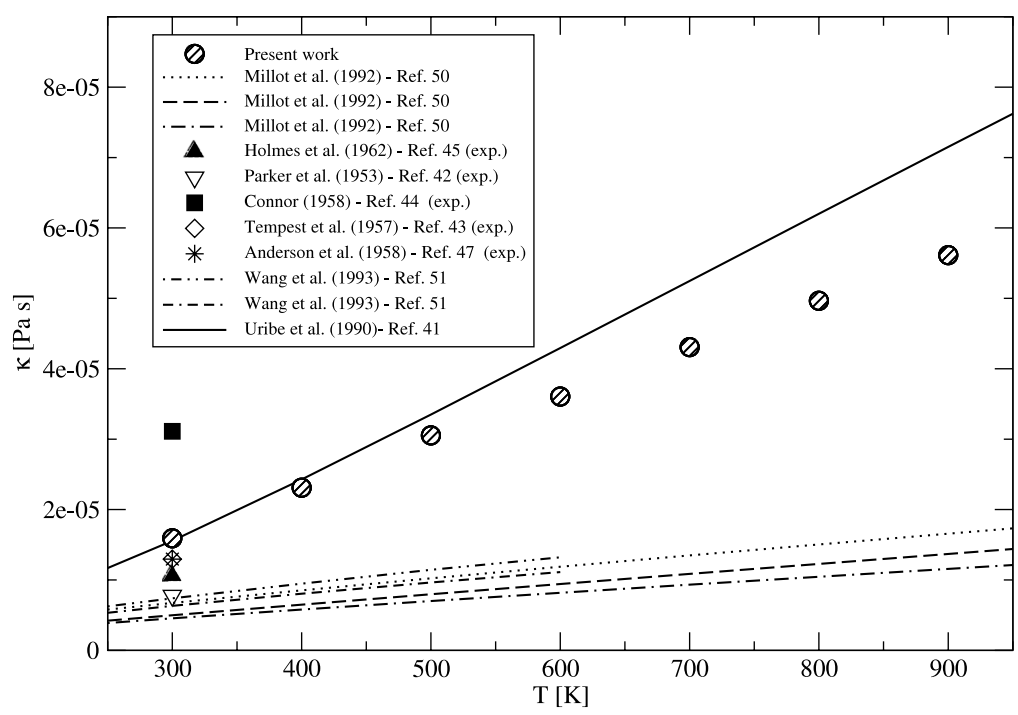

FIG. 9. $\mathrm{O}_{2}$ bulk viscosity $\kappa$ as a function of temperature. Present work estimates compared with the literature experimental data and previous estimations. Dotted line, dashed line, and dot-dashed line represent estimations based on experimental rate constants from Ref. 50. 
Rotational relaxation is also responsible of the shock thickness increase; therefore, determination of this quantity has been obtained from shock thickness measurements. ${ }^{47}$

Measurements are then reported in terms of the rotational relaxation number, $Z_{R}$, using ${ }^{2}$

$$
\kappa=\frac{k_{B}}{c_{v}} \frac{c_{i}}{c_{v}} p Z_{R} \tau_{c}
$$

where $\tau_{c}$, the mean collision time, is evaluated as

$$
\tau_{c}=\frac{\pi \eta}{4 p}
$$

In this work, we have recovered values for the bulk viscosity from the published $Z_{R}$ by using the recommended value for shear viscosity ${ }^{37}$ This choice is not critical since most available estimations of the shear viscosity have a scatter smaller than $5 \%$, and this uncertainty is much smaller than the scatter obtained for the bulk viscosity coefficient (see Fig. 9).

Most of the measurements have been made at room temperature, except for Ref. 46, where the range $T=300-1300 \mathrm{~K}$ is explored. In their critical data compilation, ${ }^{41}$ Uribe et al. recommend to use the following expression from Ref. 48:

$$
Z_{R}=\tilde{Z}\left[1+\frac{\pi \sqrt{\pi}}{2} \sqrt{\frac{\tilde{T}}{T}}+\left(\frac{\pi^{2}}{4}+2\right) \frac{\tilde{T}}{T}+\pi \sqrt{\pi}\left(\frac{\tilde{T}}{T}\right)^{\frac{3}{2}}\right]^{-1} .
$$

The expression above represents an extension of a previous work, ${ }^{49}$ where the classical dynamics for rigid rotor collisions is solved by assuming a simple form for the interaction potential. The recommended parameters for the model are determined by fitting the best sound absorption experimental data, ${ }^{46}$

$$
\tilde{Z}=36, \quad \tilde{T}=121.1 \mathrm{~K} .
$$

As shown in Fig. 9, where the predictions of Eq. (36) are represented by the solid line, the agreement with CT-MC values is good up to about $500 \mathrm{~K}$. At higher temperatures, Eq. (36) predictions are above CT-MC values. It is also to be noted that the CT-MC bulk viscosity value at $300 \mathrm{~K}$ is in good agreement with most of the experimental data. An exception is represented by the value given in Ref. 44 which is also well above all other predictions. The rotational relaxation number has also been determined by solving the classical rigid rotor dynamics from different PESs ${ }^{51-53}$ The results of Ref. 53 are presented in Fig. 9 as double-dash-dotted and double-dot-dashed lines in the temperature range $300-600 \mathrm{~K}$.

Inelastic collisions are also responsible for line mixing and line broadening effects in Raman spectra. ${ }^{50}$ Starting from high-resolution stimulated Raman spectra, researchers were able to determine the parameters for a few fitting laws modeling the inelastic rate constants. ${ }^{50}$ These fitting laws have been shown to reproduce correctly Raman spectra in different bands and in different pressure and temperature conditions..$^{50,54,55}$ They have been used here to obtain an independent estimation of bulk viscosity. To this end, only rotational degrees of freedom have been considered. The calculation is based on the relationship

$$
\kappa=\left(\frac{c_{i}}{c_{v}}\right)^{2} \frac{k_{B} T}{2\left[\left[\left(\frac{\Delta E}{k_{B} T}\right)^{2}\right]\right]}
$$

derived from Chapman-Enskog expansion. ${ }^{2}$ In Eq. (38), $c_{i}$ and $c_{v}$ are the internal and the total constant volume specific heats, respectively, equal to $k_{\mathrm{B}}$ and $5 k_{\mathrm{B}} / 3$, whereas $\Delta E$ is the energy exchanged in collisions. The symbol [[]] denotes thermal average over all collisions. The estimation of the bulk viscosity can be connected to the rates of rotational relaxation $K_{i j}^{k l}(T)$ by noting that

$$
\left[\left[\left(\frac{\Delta E}{k_{B} T}\right)^{2}\right]\right]=\frac{1}{8} \sum_{i, j, k, l}\left(\frac{\Delta E_{i j}^{k l}}{k_{B} T}\right)^{2} \frac{n_{i}}{n} \frac{n_{j}}{n} K_{i j}^{k l}(T) .
$$


Bulk viscosity values have then been obtained from Eqs. (38) and (39) by using three different fitting laws for the rates of rotational relaxation (MEG, ECS-P, and ECS-EP, see Ref. 50 for a description and related references) with parameters from Ref. 50. Results are reported in Fig. 9 as dotted, dashed, and dot-dashed lines, respectively. Theoretical calculations ${ }^{53}$ are in good agreement with the values derived from Raman spectroscopy, but they underestimate substantially the recommended values, except for low temperature. This may be caused by overestimation of the inelastic rates for high-lying rotational states.

\section{CONCLUSIONS}

A potential energy surface derived from molecular beam experiments ${ }^{8}$ has been used to describe the collisional interaction among $\mathrm{O}_{2}$ molecules, in the rigid rotator approximation. Both equilibrium and non-equilibrium CT-DSMC simulations have been used to obtain molecular oxygen transport coefficients in the temperature range $T=300-900 \mathrm{~K}$. The adopted methods provide close predictions of transport coefficients. Comparisons with the literature experimental data show good agreement, with discrepancies around $3 \%-5 \%$ for shear viscosity, i.e., below the accuracy of the data. Good agreement is also found for thermal conductivity. However, when temperature is increased, the predicted thermal conductivity exhibits small but systematically increasing deviations from experimental reference data, which can be attributed to neglecting vibrational degrees of freedom. A notable exception is the bulk viscosity coefficient that shows considerable spread in the available estimations. This points to the need of improving the current understanding of rotational relaxation for this system.

It is worth stressing that the increasing availability of accurate PESs makes CT-DSMC technique of considerable interest to explore non-equilibrium flows involving molecular species and to contribute to the development of simpler molecular collision models. Although computationally demanding, CT-DSMC can be extended to deal with simple gases or mixtures composed by non-linear molecules which can be either polar or non-polar.

\section{ACKNOWLEDGMENTS}

The authors gratefully acknowledge the support of NVIDIA Corporation within the framework of the "Hardware Donation Program."

\section{APPENDIX A: RADIAL POTENTIAL COEFFICIENTS}

This Appendix gives the form of the radial potential coefficients appearing in Eq. (1), as defined in Ref. 8.

The isotropic contribution $V^{000}(R)$ has the form of a Morse-spline-van der Waals potential, expressed in terms of rescaled quantities $x=\frac{R}{R_{m}}$ and $f(x)=\frac{V^{000}}{\epsilon_{m}}$ as

$$
f(x)= \begin{cases}\exp [-2 \beta(x-1)]-2 \exp [-\beta(x-1)], & x \leq x_{1} \\ b_{1}+(x-x 1)\left\{b_{2}+\left(x-x_{2}\right)\left[b_{3}+\left(x-x_{1}\right) b_{4}\right],\right\} & x_{1}<x \leq x_{2} \\ -\frac{C^{000}}{\epsilon_{m} R_{m}^{6}} x^{-6}, & x>x_{2}\end{cases}
$$

The values of the various constants are given in Table II. The coefficients $b_{i}, i=1, \ldots, 4$ of the cubic spline connecting the Morse to van der Waals contribution are uniquely specified by imposing continuity of $f$ and its first derivative at $x_{1}$ and $x_{2}$. 
TABLE II. Parameters' values for $V^{000}(R)$.

\begin{tabular}{lccccc}
\hline \hline$R_{m}(\AA)$ & $\epsilon_{m}(\mathrm{meV})$ & $\beta$ & $x_{1}$ & $x_{2}$ & $C^{000}(\mathrm{meV}) \AA^{6}$ \\
\hline 3.90 & 11.7 & 6.7 & 1.12 & 1.55 & $5.3 \times 10^{4}$ \\
\hline \hline
\end{tabular}

The anisotropic radial terms $V^{202}\left(R_{12}\right), V^{220}\left(R_{12}\right)$, and $V^{222}\left(R_{12}\right)$ are assumed to have the forms

$$
\begin{aligned}
& V^{202}(R)=A^{202} \exp \left(-c^{202} R-d^{202} R^{2}\right)-\frac{C^{202}}{R^{6}}, \\
& V^{220}(R)=A^{220} \exp \left(-c^{220} R\right), \\
& V^{222}(R)=A^{222} \exp \left(-c^{222} R\right) .
\end{aligned}
$$

Parameters' values for these terms are given in Table III.

\section{APPENDIX B: TRANSPORT COEFFICIENTS OF DIATOMIC GASES FROM CHAPMAN-ENSKOG EXPANSION}

The following expressions, derived by Taxman in Ref. 10 and reported here for completeness sake, provide the first approximation ${ }^{2}$ of transport coefficients of a gas composed by diatomic rigid molecules.

Shear viscosity,

$$
\frac{1}{\eta}=\frac{8}{5 \sqrt{\pi m_{O_{2}} k_{\mathrm{B}} T}} \Phi^{-2} \int\left[\gamma^{4} \sin ^{2} \chi-\left(\frac{2}{3} \gamma^{\prime 2}+\gamma^{2} \sin \chi\right) \Delta \epsilon\right] e^{-\gamma^{2}-\epsilon_{1}-\epsilon_{2}} \gamma^{3} b d b d \phi d \gamma d \Omega_{1} d \Omega_{2} .
$$

Bulk viscosity,

$$
\frac{1}{\kappa}=\frac{10}{\sqrt{\pi m_{O_{2}} k_{\mathrm{B}} T}} \Phi^{-2} \int\left[\left(3 \epsilon_{1}^{\prime}-\frac{3}{2}-\gamma^{2}\right) \Delta \epsilon+\Delta \epsilon^{2}\right] e^{-\gamma^{2}-\epsilon_{1}-\epsilon_{2}} \gamma^{3} b d b d \phi d \gamma d \Omega_{1} d \Omega_{2} .
$$

Thermal conductivity,

$$
\lambda=\frac{3}{2} \frac{k_{\mathrm{B}}}{m_{O_{2}}} \sqrt{\pi m_{O_{2}} k_{\mathrm{B}} T}\left[X-\frac{5}{2}(Y+\tilde{Y})+\frac{25}{4} W\right](X W-Y \tilde{Y})^{-1},
$$

where

TABLE III. Parameters' values for $V^{202}(R), V^{220}(R)$, and $V^{222}(R)$.

\begin{tabular}{lccc}
\hline \hline$A^{202}(\mathrm{meV})$ & $c^{202}\left(\AA^{-1}\right)$ & $d^{202}\left(\AA^{-2}\right)$ & $C^{202}\left(\mathrm{meV}^{6}\right)$ \\
\hline $7.7 \times 10^{6}$ & 4.0 & -0.060 & $6.12 \times 10^{3}$ \\
\hline$A^{220}(\mathrm{meV})$ & $V^{220}$ & \\
\hline $5.0 \times 10^{6}$ & & $c^{220}\left(\AA^{-1}\right)$ \\
\hline$A^{222}(\mathrm{meV})$ & $V^{222}$ & 3.83 \\
\hline$-4.0 \times 10^{6}$ & & $c^{222}\left(\AA^{-1}\right)$ \\
\hline \hline
\end{tabular}




$$
\begin{gathered}
X=4 \Phi^{-2} \int\left[\gamma^{4} \sin ^{2} \chi+\left(\gamma^{2} \cos ^{2} \chi+\frac{25}{8}-\frac{15}{4} \gamma^{2}\right)+\frac{11}{4} \Delta \epsilon^{2}\right] e^{-\gamma^{2}-\epsilon_{1}-\epsilon_{2}} \gamma^{3} b d b d \phi d \gamma d \Omega_{1} d \Omega_{2} \\
Y=-5 \Phi^{-2} \int\left[\left(\frac{3}{2}-\gamma^{2}\right) \Delta \epsilon+\Delta \epsilon^{2}\right] e^{-\gamma^{2}-\epsilon_{1}-\epsilon_{2}} \gamma^{3} b d b d \phi d \gamma d \Omega_{1} d \Omega_{2} \\
\tilde{Y}=4 \Phi^{-2} \int\left(\epsilon_{1}^{\prime}-1\right) \Delta \epsilon e^{-\gamma^{2}-\epsilon_{1}-\epsilon_{2}} \gamma^{3} b d b d \phi d \gamma d \Omega_{1} d \Omega_{2} \\
W=-10 \Phi^{-2} \int\left(\epsilon_{1}^{\prime}-1\right)\left[\frac{3}{2} \Delta \epsilon+\gamma^{\prime 2}\left(\epsilon_{1}^{\prime}-\epsilon_{2}^{\prime}\right)-\gamma \gamma^{\prime}\left(\epsilon_{1}-\epsilon_{2}\right) \cos \chi\right] e^{-\gamma^{2}-\epsilon_{1}-\epsilon_{2}} \gamma^{3} b d b d \phi d \gamma d \Omega_{1} d \Omega_{2} .
\end{gathered}
$$

In the expression above, $\gamma=\left(4 k_{\mathrm{B}} T / m_{O_{2}}\right)^{-1 / 2} g$, being $g$ the absolute value of the relative precollision velocity of the particles, $\epsilon$ the internal energy, and $\chi$ the deflection angle. Quantities with superscript refer to post-collision state and $\Delta \epsilon=\epsilon_{1}^{\prime}+\epsilon_{2}^{\prime}-\epsilon_{1}-\epsilon_{2}=\gamma^{2}-\gamma^{\prime 2}$ represents the variation of the internal energy due to a collision. $\Phi=\int e^{-\epsilon} d \Omega$. The well-known Chapman-Enskog results for a monatomic gas ${ }^{2}$ are recovered by setting $\Delta \epsilon$ and the rotational constant volume specific heat $c_{i}$ equal to zero.

${ }^{1}$ C. Cercignani, The Boltzmann Equation and Its Applications (Springer-Verlag, Berlin, 1988).

2 J. H. Ferziger and H. G. Kaper, Mathematical Theory of Transport Processes in Gases (North-Holland, 1972).

${ }^{3}$ F. Sharipov and J. L. Strapasson, "Direct simulation Monte Carlo method for an arbitrary intermolecular potential," Phys. Fluids 24, 011703 (2012).

${ }^{4}$ S. Chapman and T. G. Cowling, The Mathematical Theory of Non-Uniform Gases (Cambridge University Press, Cambridge, UK, 1990).

${ }^{5}$ G. A. Bird, Molecular Gas Dynamics and the Direct Simulation of Gas Flows (Clarendon Press, Oxford, 1994).

${ }^{6}$ C. Borgnakke and P. S. Larsen, "Statistical collision model for Monte Carlo simulation of polyatomic gas mixtures," J. Comput. Phys. 18, 405-420 (1975).

${ }^{7}$ I. J. Wysong and D. C. Wadsworth, "Assessment of direct simulation Monte Carlo phenomenological rotational relaxation models," Phys. Fluids 10, 2983-2994 (1998).

${ }^{8}$ V. Aquilanti, D. Ascenzi, M. Bartolomei, D. Cappelletti, S. Cavalli, M. de Castro Vitores, and F. Pirani, "Molecular beam scattering of aligned oxygen molecules. The nature of the bond in the $\mathrm{O}_{2}-\mathrm{O}_{2}$ dimer," J. Am. Chem. Soc. 121, 10794-10802 (1999).

${ }^{9}$ W. Cencek, K. Szalewicz, C. Leforestier, R. van Harrevelt, and A. van der Avoird, "An accurate analytic representation of the water pair potential," Phys. Chem. Chem. Phys. 10, 4716-4731 (2008).

${ }^{10}$ N. Taxman, "Classical theory of transport phenomena in dilute polyatomic gases," Phys. Rev. 110, 1235-1239 (1958).

${ }^{11}$ F. R. W. McCourt, J. J. M. Beenakker, W. E. Köhler, and I. Kuscer, Nonequilibrium Phenomena in Polyatomic Gases, International Series of Monographs on Chemistry Vol. 18 (Oxford University Press, 1990).

12 J. A. Lordi and R. E. Mates, "Rotational relaxation in nonpolar diatomic gases," Phys. Fluids 13, 291-308 (1970).

${ }^{13}$ R. Hellmann, E. Bich, E. Vogel, A. S. Dickinson, and V. Vesovic, "Calculation of the transport and relaxation properties of dilute water vapor,’ J. Chem. Phys. 131, 014303 (2009).

${ }^{14}$ R. Hellmann, E. Bich, E. Vogel, A. S. Dickinson, and V. Vesovic, "Calculation of the transport and relaxation properties of methane. I. Shear viscosity, viscomagnetic effects, and self-diffusion,” J. Chem. Phys. 129, 064302 (2008).

${ }^{15}$ M. A. ter Horst and C. J. Jameson, “A comparative study of $\mathrm{CO}_{2}-\mathrm{Ar}$ potential surfaces," J. Chem. Phys. 105, 6787-6806 (1996).

${ }^{16}$ K. Koura, "Monte Carlo direct simulation of rotational relaxation of diatomic molecules using classical trajectory calculations: Nitrogen shock wave," Phys. Fluids 9, 3543-3549 (1997).

${ }^{17}$ K. Koura, "Direct simulation Monte Carlo study of rotational nonequilibrium in shock wave and spherical expansion of nitrogen using classical trajectory calculations," Phys. Fluids 14, 1689-1695 (2002).

${ }^{18}$ P. Norman, P. Valentini, and T. Schwartzentruber, "GPU-accelerated classical trajectory calculation direct simulation Monte Carlo applied to shock waves," J. Comput. Phys. 247, 153-167 (2013).

19 D. Ferrigni, "Simulation of rotational relaxation in diatomic gas by classical trajectories on GPU's," M.S. thesis (Politecnico di Milano, 2012).

${ }^{20}$ J. P. Boon and S. Yip, Molecular Hydrodynamics, Dover Books on Physics (Dover Publications, Inc., New York, 1980).

${ }^{21}$ M. Abramowitz and I. Stegun, Handbook of Mathematical Functions, Dover Books on Mathematics (Dover, 1965).

${ }^{22}$ M. Allen and D. Tildesley, Computer Simulation of Liquids (Clarendon Press, Oxford, 1987).

${ }^{23}$ C. F. Curtiss, "The classical Boltzmann equation of a gas of diatomic molecules," J. Chem. Phys. 75, 376-378 (1981).

${ }^{24}$ R. Kubo, M. Toda, and N. Hashitsume, Statistical Physics II (Springer, 1985).

${ }^{25}$ D. J. Evans and G. Morriss, Statistical Mechanics of Nonequilibrium Liquids (Cambridge University Press, 2008).

${ }^{26}$ A. J. H. McGaughey and M. Kaviany, "Quantitative validation of the Boltzmann transport equation phonon thermal conductivity model under the single-mode relaxation time approximation," Phys. Rev. B 69, 094303 (2004).

27 D. Bruno and V. Giovangigli, "Relaxation of internal temperature and volume viscosity," Phys. Fluids 23, 093104 (2011).

${ }^{28}$ M. Frigo and S. G. Johnson, "The design and implementation of FFTW3," Proc. IEEE 93, 216-231 (2005). 
${ }^{29}$ A. Panarese, D. Bruno, G. Colonna, P. Diomede, A. Laricchiuta, S. Longo, and M. Capitelli, "A Monte Carlo model for determination of binary diffusion coefficients in gases," J. Comput. Phys. 230, 5716-5721 (2011).

${ }^{30}$ R. D. Present, Kinetic Theory of Gases (McGraw-Hill, New York, 1958).

${ }^{31}$ M. J. Wright, D. Bose, G. E. Palmer, and E. Levin, "Recommended collision integrals for transport property computations part 1: Air species," AIAA J. 43, 2558-2564 (2005).

32 J. Bzowski, J. Kestin, E. A. Mason, and F. J. Uribe, "Equilibrium and transport properties of gaseous mixtures at low density: Eleven polyatomic gases and five noble gases," J. Phys. Chem. Ref. Data 19, 1179-1231 (1990).

${ }^{33}$ C. Cercignani and S. Cortese, "Validation of a Monte Carlo simulation of the plane Couette flow of a rarefied gas," J. Stat. Phys. 75, 817 (1994)

${ }^{34}$ A. Frezzotti, "Monte Carlo simulation of the uniform shear flow in a dense rough sphere fluid," Physica A 278, 161-180 (2000).

${ }^{35}$ V. Garzó and A. Santos, Kinetic Theory of Gases in Shear Flows (Springer, 2003).

36 J. M. Montanero and A. Santos, "Viscosimetric effects in a dense hard sphere fluid," Physica A 240, 229-238 (1997).

${ }^{37}$ E. W. Lemmon and R. T. Jacobsen, "Viscosity and thermal conductivity equations for nitrogen, oxygen, argon, and air," Int. J. Thermophys. 25, 21-69 (2004).

${ }^{38}$ J. Montanero, M. Alaui, A. Santos, and V. Garzó, "Monte Carlo simulation of the Boltzmann equation for steady Fourier flow," Phys. Rev. E 49, 367-375 (1994).

${ }^{39}$ M. A. Gallis, J. R. Torczynski, D. J. Rader, M. Tij, and A. Santos, "Normal solutions of the Boltzmann equation for highly nonequilibrium Fourier flow and Couette flow," Phys. Fluids 18, 017104 (2006).

${ }^{40}$ D. J. Rader, M. A. Gallis, J. R. Torczynski, and W. Wagner, "Direct simulation Monte Carlo convergence behavior of the hard-sphere-gas thermal conductivity for Fourier heat flow," Phys. Fluids 18, 077102 (2006).

${ }^{41}$ F. J. Uribe, E. A. Mason, and J. Kestin, "Thermal conductivity of nine polyatomic gases at low density," J. Phys. Chem. Ref. Data 19, 1123-1136 (1990).

42 J. G. Parker, C. E. Adams, and R. M. Stavseth, "Absorption of sound in argon, nitrogen, and oxygen at low pressures," J. Acoust. Soc. Am. 25, 263 (1953).

${ }^{43}$ W. T. Tempest and H. D. Parbrook, "The absorption of sound in argon, nitrogen and oxygen," Acta Acust. United Ac. 7 , 354-362 (1957).

44 J. V. Connor, “Ultrasonic dispersion in oxygen,” J. Acoust. Soc. Am. 30, 297 (1958).

${ }^{45}$ R. Holmes, G. R. Jones, N. Pusat, and W. Tempest, "Rotational relaxation in helium + oxygen and helium + nitrogen mixtures," Trans. Faraday Soc. 58, 2342-2347 (1962).

${ }^{46}$ E. H. Carnevale, C. Carey, and G. Larson, "Ultrasonic determination of rotational collision numbers and vibrational relaxation times of polyatomic gases at high temperatures," J. Chem. Phys. 47, 2829 (1967).

${ }^{47}$ W. H. Anderson and D. F. Hornig, Technical Report No. 8, Metcalf Research Laboratory, Brown University, 1958.

${ }^{48}$ C. A. Brau and R. Jonkman, "Classical theory of rotational relaxation in diatomic gases," J. Chem. Phys. 52, 477 (1970).

49 J. G. Parker, "Rotational and vibrational relaxation in diatomic gases," Phys. Fluids 2, 449-462 (1959).

${ }^{50}$ G. Millot, R. Saint-Loup, J. Santos, R. Chaux, H. Berger, and J. Bonamy, "Collisional effects in the stimulated Raman Q branch of $\mathrm{O}_{2}$ and $\mathrm{O}_{2}-\mathrm{N}_{2}$, , J. Chem. Phys. 96, 961-971 (1992).

${ }^{51}$ A. F. Turfa, H. P. F. Knaap, B. J. Thijsse, and J. J. M. Beenakker, "A classical dynamics study of rotational relaxation in nitrogen gas," Physica A 112, 18-28 (1982).

${ }^{52}$ C. Nyeland, L. L. Poulsen, and G. D. Billing, "Rotational relaxation and transport coefficients for diatomic gases: Computations on nitrogen," J. Phys. Chem. 88, 1216 (1984).

${ }^{53}$ L. Wang and G. D. Billing, "Rotational relaxation and transport properties of oxygen by using the importance sampling method," J. Phys. Chem. 97, 2523-2526 (1993).

${ }^{54}$ T. Dreier, G. Schiff, and A. A. Suvernev, "Collisional effects in Q branch CARS-spectra of $\mathrm{N}_{2}$ and $\mathrm{O}_{2}$ at high pressure and high temperature," J. Chem. Phys. 100, 6275 (1994).

${ }^{55}$ H. Tran, C. Boulet, and J.-M. Hartmann, "Line mixing and collision-induced absorption by oxygen in the A band: Laboratory measurements, model, and tools for atmospheric spectra computations," J. Geophys. Res. 111, D15210 doi:10.1029/2005JD006869 (2006). 